\title{
10 Local Brokerage Positions and Access to Unique Information
}

\begin{abstract}
The theory of structural holes (Burt 1995) provides an explanation regarding network positions and their associated benefits in many social settings. One of the key points of the theory is formulated on the node level and is about efficient access to information and resources. Namely, actors bridging structural holes by connecting otherwise disconnected segments of a social network, the brokers, have access to information that circulates over the network while simultaneously maintaining relatively small number of ties. For example, scientists often maintain non-redundant collaboration ties because they are associated with academically-relevant resources unavailable in other collaborations. Apart from the above node-level property one can formulate a tie-level property corresponding to the 'tie redundancy'. Studying a process of the network information diffusion explicitely with a computational experiment and tools of information theory allows us to compare local properties of the diffusion with structural features related to the Burtian notions of brokerage and redundancy. We argue and demonstrate that (1) the above mentioned node-level and tie-level properties can be supplemented with a triad-level one. Specifically, that the notion of 'tie redundancy' should be in fact considered as an essentially triadic property. Further, (2) each of the three properties is associated with a viable empirical strategy of testing whether (aspects of) the structural holes mechanism is at work in a given network.
\end{abstract}

\subsection{Introduction}

The theory of structural holes (Burt 1995) identifies network positions having ties that connect otherwise unconnected segments of a social network as brokerage positions. Occupying a brokerage position in social networks is usually associated with various benefits. Further, the theory stipulates, among other things, that it is beneficial for an actor to occupy such broker positions as it allows for efficient access to information that spreads in the network. This statement pretends to the node level - it specifies that certain actors (network nodes), namely brokers, will be more successful than other actors given some appropriate notion and measure of success. For example, managers occupying such positions in an organization have been shown to be more likely to be promoted (Burt, Hogarth, and Michaud 2000).

Note: Author thanks Polish National Science Centre (NCN) for support for the RECON project (http://recon.icm.edu.pl/) through grant 2012/07/D/HS6/01971.

Michat Bojanowski, Kozminski University

Ә Open Access. (C) 2020 Michat Bojanowski, published by De Gruyter. (c) BY-NC-ND This work is licensed under a Creative Commons Attribution-NonCommercial-NoDerivatives 4.0 International License.

https://doi.org/10.1515/9783110647495-010 
Among the other elements of the theory of structural holes is the one about information flow and access to resources. It is related to the notion of tie redundancy. A tie is redundant for an actor to an extent that it connects others, to whom an actor already has access via his other ties. This concept is related to the concept of weak ties (Granovetter 1977). Granovetterian "weak ties”, or Burtian "non-redundant” ties, are usually sources of information that is less likely to be acquired through "strong" or "redundant" ties. As such, it is a statement on the tie level. It has been famously shown by Granovetter (1974) that people usually learned about their current job through social contacts who are not well embedded in their personal networks. ${ }^{1}$

When approaching a research setting with a question whether the structural holes mechanism is at work one usually tests one of the two implications mentioned above: whether brokers are more succesful than non-brokers or whether relevant information is usually coming to an actor through a non-redundant rather than a redundant tie. Consider Figure 10.1 presenting a hypothetical personal network of actor $A$. We assume that this personal network is embedded in a much larger network alters of $A$ might have further connections, but we do not know them. We can (1) mark through which actor $A$ learned the information of interest (e.g. about a job), and (2) measure the extent of redundancy of each of $A$ 's ties, e.g. using dyadic redundancy. If the tie-level implication of structural holes is true the information is more likely to come to $A$ from alters with low redundancy. Most likely actor $F$.

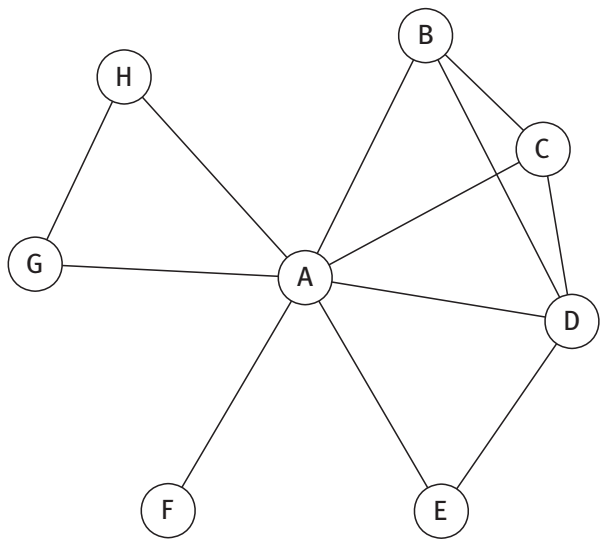

Figure 10.1: For actor $A$ alters $G, H$, and $E$ are redundant to the same extent. At the same time, sets of actors G-H and B-C-D-E can be sources of different information as they are disconnected from each other.

1 While the issues addressed in this paper can be argued to operate on a scale of complete, as opposed to ego-centric, graphs (see Borgatti 2005) we are limiting our attention to personal networks. This is of great relevance as a lot of empirical studies of the mentioned phenomena, including the seminal work of Granovetter (1974), is conducted with ego-centrically sampled network data. 
We argue that the abovementioned approach is often insufficient. Consider a research setting in which there are multiple types of information circulating in the social network and none of these types is any way more special than other types. For example, collaboration between scientists is often motivated by a possibility to acquire or exchange various resources relevant for functioning in academia, such as specific expertise, access to costly equipment or mentorship. ${ }^{2}$ As all types of information are equally salient we cannot approach the problem, as suggested by the tie-level implication, by studying a probability of receiving "new" information as a function of tie redundancy. None of the information is "new". What we need instead is to analyze whether the information received through a non-redundant tie is different from the information recieved through the other ties. In Figure 10.1 actors $E, G$, and $H$ are redundant for the actor $A$ to the same extent because each has exactly one tie to other contacts of $A$. However, they are clearly not redundant vis a vis each other. For example, the lack of connection between $E$ and $H$ might suggest that they can be sources of different information even though they are redundant to the same extent. What we need then is triadic notion of redundancy and a way of measuring how the information coming to $A$ from $E$ might be different from that coming to $A$ from $H$.

To achieve that we, first, in Section 10.2, develop qualitatively the implications of Burtian theory of structural holes for node-, tie- and triad-level properties of information passing through personal networks of actors. Second, we formalize the process of information flow as a stochastic process in Section 10.3. Third, in order to study these properties we designed a computer simulation described in Section 10.4. Among the results presented in Section 10.5 are five conjectures relating, on the one hand, local properties of the information flow and structural characteristics of the ego-network on the other hand. In particular, the developed a triad-level implication of the theory of structural holes together with the simulation results provide a stronger theoretical justification for the index of pairwise redundancy proposed by Bojanowski and Czerniawska-Szejda (2018). We conclude the paper with Section 10.6 by discussing, among other things, what empirical strategies the presented results suggest that are applicable in research settings in which there are multiple types of resources/information being shared through a social network.

\subsection{Brokerage and information flow}

Actors facing a network information diffusion process can have interests in various aspects of such a situation. Important instance that we will focus on is access to

2 We might expect, as argued by Bojanowski and Czerniawska-Szejda (2018), that structurally nonredundant collaboration ties will be associated with resources that a scientist is unable to acquire through his other collaborations. 
information, that is staying informed. An actor might be interested in maximizing chances that any information appearing somewhere in the network will eventually reach him. Other examples include being informed as soon as possible (Buskens and Yamaguchi 1999; Buskens 2002, chap. 4) or maintaining power defined as control over information flow (Reagans and Zuckerman 2008; Emerson 1962). If staying informed is assumed to be a goal motivating tie-forming and tie-dissolving actions of the actors the question of what kind of social networks will emerge endogenously via actors' relational choices becomes a micro-macro problem in the sense of Raub, Buskens, and Van Assen (2011). An example is depicted in Figure 10.2. Macro conditions include assumed properties of the process of information spreading in the network. Properties such as: whether actor loses information when it is shared or not, how the probability of some actor $i$ passing information to some actor $j$ is defined. Micro conditions include assumptions about the goals and constraints of the actors such as willingness of staying informed while maintaining as few connections as possible. Should the theory of structural holes apply, actors will be likely to drop structurally redundant ties as they are a liability. This would be a micro-level outcome. Removal of redundant ties by the actors leads to appearance of brokers on the macro level. Further, Buskens and Van de Rijt (2008) shown that the value of brokerage positions lies in a "social opportunity structure" that is exhaustible.

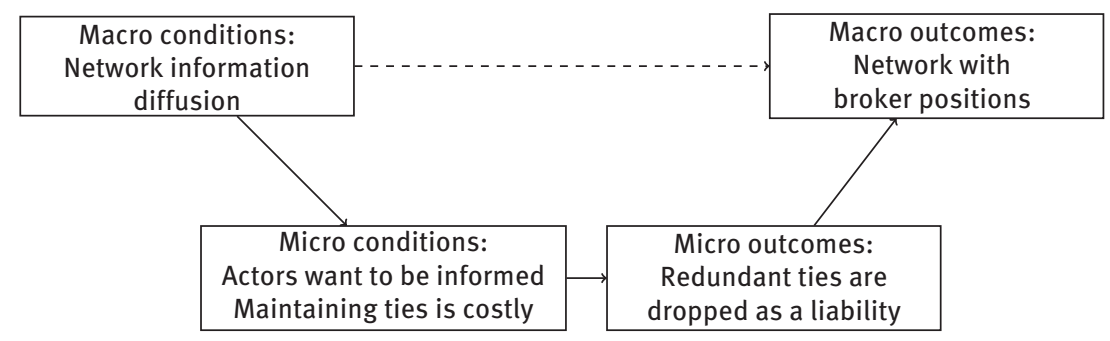

Figure 10.2: Micro-macro mechanism of emergence of brokers when facing information diffusion.

Empirically, when analyzing a single snapshot of a network we usually observe a mixture of broker and non-broker positions. This can be because of the network being far from the structural hole-less equilibrium derived by Buskens and Van de Rijt (2008) or there might be other network formation incentives operating apart from brokerage.

As signaled in the Section 1, brokerage and its properties can be analyzed on different levels: nodes, ties, and triads:

- Nodes: Position of a broker in an information flow can be expected to be "information-efficient". That is, every tie should serve a purpose in the sense of being a channel for specific information unavailable through other ties. Statistically 
speaking the information type arriving to an actor with high brokerage should be correlated with (predictable from) the alter that it is arriving from.

- Ties: Information coming to an actor through a non-redundant tie should be different from that coming through other ties. In statistical terms the distribution of information type coming through a non-redundant tie should be different from the distribution of information type arriving through all the other ties combined.

- Triads: Not all redundant ties are alike, even if they are redundant to the same extent. As exemplified with actors $E$ and $H$ in Figure 10.1, an alter can be more or less redundant to ego vis a vis some other alter. In other words, it is a triadic property. We can expect that pairs of alters who are, for example, disconnected in ego's neighborhood to be sources of more different information than pairs of alters who are connected. In statistical terms the distributions of information type in those two pairs will be more similar for connected than for disconnected pairs of alters.

Concrete statistical tools to quantify above mentioned concepts such as brokerage, dyadic and triadic redundancy, as well as for measuring dependence and (dis)similarity of distributions will be introduced in detail in Section 10.5 below.

\subsection{The model of information diffusion}

Let us have an undirected network $G=\{V, E\}$. Every actor $s \in V$ is endowed with a unique piece of information which will be spreading through the network. At any given time every actor $i \in V$ can be either informed or uninformed about information $s$. Actor $i$ is informed about $s$ if the information that started spreading from actor $s$ has reached him in the past. He is uninformed about $s$ otherwise. As every actor is an origin of unique information his identity ( $s$ ) defines the "information type" - if some actor $i$ learns the information that started spreading from some other actor $s$ we might say that actor $i$ learned information type $s$. The overall history of information status of all actors can be described with an array $M=\left[m_{i s t}\right]$ such that an element $m_{\text {ist }}=1$ if at time $t$ actor $i$ is informed about information $s$ and $m_{i s t}=0$ otherwise.

In principle the diffusion processes of different types of information may interact. For example, consider some actor $i$ informed about information types $s$ and $s^{\prime}$. The probabilities of sharing $s$ and $s^{\prime}$ with some neighbor $j$ of $i$ may not be independent. Actor $i$ might share $s$ or $s^{\prime}$ at a given time but not both. In this paper we restrict our attention to models in which the processes of diffusion of different types of information are independent. That is, the probability that actor $i$ shares information $s$ with actor $j$ does not depend on whether $i$ is informed or uninformed about some other information type $s^{\prime}$, whether he spread $s^{\prime}$ to $j$ at the same time step or in the past, etc. 
Information spreads through network ties at discrete times. This can be modeled in several ways. For example, Buskens and Yamaguchi (1999) consider two scenarios:

1. the multinomial version in which such information-passing events are independent between actors. The two actors can spread information each to a single neighbor at a single time step

2. the product-binomial version in which information-sharing events are additionally independent within actors. A single actor can spread the information to multiple neighbors (see also Buskens 2002, chap. 4).

Similarly, Borgatti (2005) considers a "gossip model” which is similar to scenario (1) above, but with additional restrictions. First, at each time step the information is shared once. Second, an actor will not share the information with a neighbor who already knows it. There is also a subtle difference in the way Borgatti (2005) implemented the model in their simulations, which we will comment upon in Section 10.4.

The model we analyze in this paper is closest to the Gossip model of Borgatti (2005). We assume that at discrete times an informed actor having at least one uninformed neighbor is selected randomly. She then informs a randomly selected uninformed neighbor. Such processes for each information type $s$ take place independently from each other. Following Buskens and Yamaguchi (1999) and Buskens (2002, chap. 4) for the sake of comparability we represent the model as a finite Markov chain.

Let $A_{i j}$ be the adjacency matrix of graph $G$ and the set of actors who are currently informed as $S_{1}$, itself a subset of $V$. Let us consider the probability that in the next step certain actor $u$ will be informed by somebody from $S_{1}$. In other words that in the next time step the set of informed actors becomes $S_{2}$ such that $S_{1} \subset S_{2}$ and $S 2 \backslash S 1=U$ where $U=\{u\}$. Define

$$
r_{i j}=\frac{A_{i j}}{\sum_{j \in V \backslash S_{1}} A_{i j}}
$$

which is a partially-normalized form of the graph adjacency matrix $A$. Of our interest will be only a submatrix of $r_{i j}$ for $i \in S_{1}$ and $j \in V \backslash S_{1}$. Elements of that submatrix are conditional probabilities that uninformed actor $j$ will be informed by $i$ given that $i$ is to inform anybody. Additionally, let $r_{i+}=\sum_{j \in V \backslash S_{1}} r_{i j}$ are row sums of that submatrix and $n_{i}$ is the number of elements of $r_{i+}$ which are positive. With that we can define the transition probability of the Markov chain as

$$
P\left(S_{1} \rightarrow S_{2}\right)= \begin{cases}0 & \text { if } S_{2} \notin S_{1} \text { or }|U| \neq 1 \\ \sum_{i: i \in S_{1} \wedge r_{i+}>0} \frac{r_{i u}}{n_{i}} & \text { otherwise }\end{cases}
$$

The chain is defined over the power set of nodes of graph $G$. 
Consider an example network from Figure 10.3. Calculating all transition probabilities leads to a process shown in Figure 10.4. It is worth noting two facts about this stochastic process:

1. There is a single absorbing state corresponding to the situation in which all actors are informed, namely $\{1,2,3,4\}$.

2. All other states constitute singleton transient sets each. In words, each of these states is visited at most once.

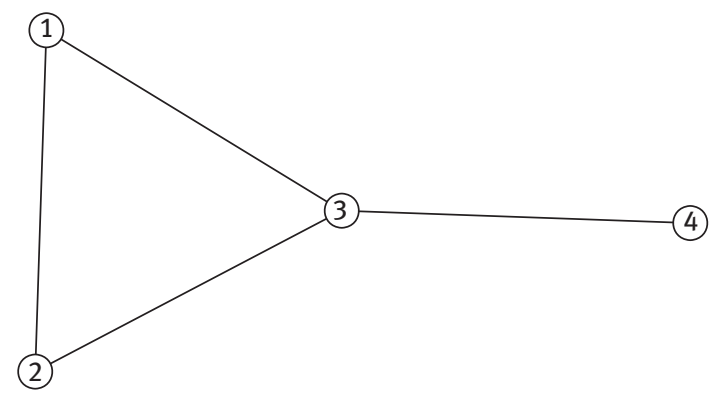

Figure 10.3: Example graph.

To compare alters with respect to the information they bring to ego we need to assess how frequently ego receives particular type of information through a particular alter. We are interested in conditional probabilities of actor $i$ informing actor $j$ with information of type $s$ conditional on actor $j$ being informed about $s$. In other words, what proportion of times actor $j$ becomes informed about $s$ through neighbor $i$ but not any other neighbor. It does not seem possible to derive such probabilities from the Markov chain defined above. One of the reasons for that difficulty is that defining the state of the chain as the set of informed actors does not provide enough detail on the dynamics of the process which would allow for calculating above mentioned conditional probabilities. To illustrate this, consider the process in the state $\{1,3\}$ and transitioning to the state $\{1,2,3\}$. In such transition actor 2 is getting informed actor 1 or actor 3 . We know from above calculation that the transition probability is 0.75 - given that actors 1 and 3 are informed in the next step actor 2 will be informed (with probability 0.75 ) or actor 4 will be informed (with probability 0.25). What we do not know is whether actor 2 learned the information from actor 1 or from actor 3. The conditional probabilities of these two events are not equal:

- Given that actor 1 is to inform anybody, she will inform actor 2 with probability 1

- Given that actor 3 is to inform anybody, she will inform actors 2 and 4 each with probability 0.5 


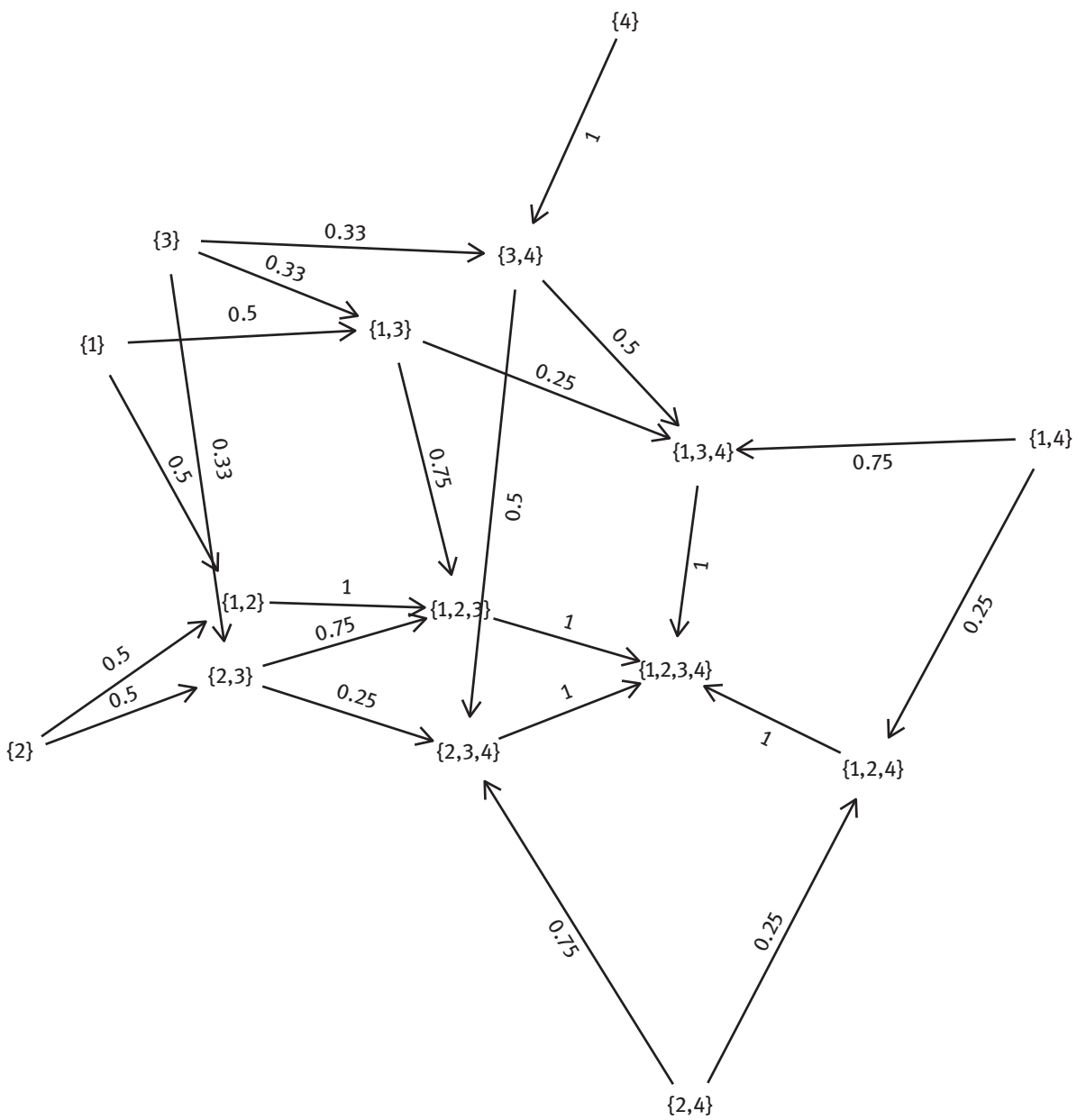

Figure 10.4: Markov Chain of information diffusion over the example graph. Each state (graph node) is a subset of actors who are informed. Arcs of the graph are state transitions labelled with transition probabilities. The set $\{1,2,3,4\}$ is an absorbing state.

Assuming that a random informed actor is to inform somebody, we select one actor from actors 1 and 3 at random. It then follows that:

- Actor 3 will inform actor 4 with probability $0.5 \cdot 0.5=0.25$.

- Actor 3 will inform actor 2 with probability $0.5 \cdot 0.5=0.25$.

- Actor 1 will inform actor 1 with probability 0.5 .

Such calculations of conditional probabilities become more complex in a generic case and on a larger graph. We therefore turn to a numerical simulation to approximate them. This is presented in the next section. 


\subsection{Simulation study}

We designed a computational experiment in which a single run consists of the following steps:

1. Take an undirected connected graph $G=\{V, E\}$.

2. Define a node attribute partitioning nodes into informed and uninformed. Initially all nodes are uninformed.

3. Select a node, called seed, and set him as informed.

4. Time progresses in discrete steps. In every time step the information is shared by a random informed node with a random network neighbor who is uninformed. In particular:

a) Identify ties that connect an informed node and an uninformed node

b) Identify informed nodes incident on the ties listed in (a)

c) Select a random node from those found in (b)

d) The informed node identified in (c) shares the information with a random uninformed neighbor.

5. Repeat (4) until all the nodes are informed.

The fact the network is connected guarantees that every node will ultimately learn the information. The design of the information diffusion model implemented in step 4 above additionally guarantees that there are no "idle" simulation steps as on every step some informed node will share the information with an uninformed node. In consequence, each simulation run always consists of exactly $|V|-1$ steps. The process is almost identical to the "gossip process" used by Borgatti (2005). The only difference is in the stopping criterion (item 5 above). Primarily for the purpose of comparability with other types of network flow processes Borgatti (2005) used a setup in which a random seed and "target" nodes were selected. The simulation was complete when starting from a single informed "source" node the process reached the state in which the "target" node became informed.

For the purpose of this paper we use the Zachary's Karate Club network (Zachary 1977). It consists of 34 nodes and 78 ties presented in Figure 10.5. We run ${ }^{3}$ the simulation 100 times for every seed node amounting to the total of $34 \times 100 \times 33=112200$ information-sharing events.

The data generated by the simulation consists of a set of information sharing events each being described with the following variables:

- $B$ - simulation run with values from $\{1, \ldots, 100\}$

- $S$ - ID of the seed actor with values from $V=\{1, \ldots, 34\}$

- $I$ - ID of the informed actor (information sender) with values from $V=\{1, \ldots, 34\}$

3 The simulation was implemented using R (R Core Team 2019) and packages: "igraph” (Csardi and Nepusz 2006), “netflow” (Bojanowski 2019), "graphlayouts” (Schoch 2019). 
- $J$ - ID of the uninformed actor (information receiver) with values from $V=\{1, \ldots, 34\}$

- $\quad T$ - Time step at at which actor $I$ shared information $S$ with actor $J$. A number from $\{1, \ldots, 33\}$.

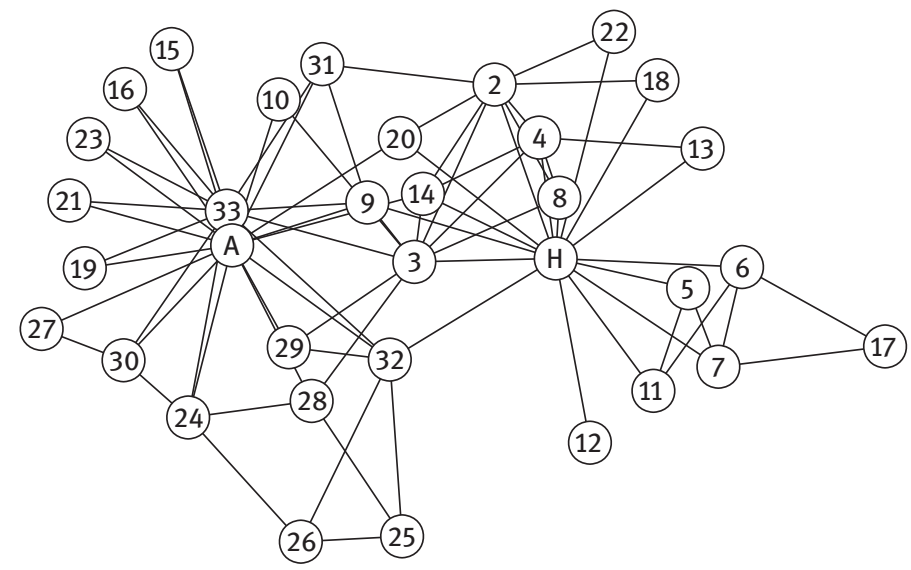

Figure 10.5: Zachary's Karate Club network used in simulations.

In words, every row is an event that took place in simulation run $B$ at time $T$ in which the information that started spreading from seed actor $S$ was passed by actor $I$ to actor $J$.

\subsection{Simulation results}

To analyze the simulated data, we are interested in studying statistical relationships between structural characteristics of personal networks on the one hand and local properties of the information flow on the other. Following the arguments from Sections 10.1 and 10.2 we discuss the results in the following subsections split by the level of analysis: actors, ties (dyads), and triads respectively.

\subsubsection{Actors}

Our intuitions from Section 10.2 were that personal networks of actors in brokerage positions will be characterized with a kind of "information efficiency" in which the message type is highly correlated with the identity of the alter through whom the actor learns it. To quantify this intuition we need measures for both concepts. Popular 
ways of capturing the extent of brokerage in a personal network is Constraint (Burt 1995, 54) defined as

$$
c_{i} \equiv \sum_{j \neq i}\left(A_{i j}+\sum_{k \neq i, k \neq j} A_{i k} A_{k j}\right)^{2}
$$

where $A$ is, as above, the adjacency matrix of the graph. Another one is network efficiency (Borgatti 1997) defined as:

$$
e_{i} \equiv \frac{1}{n}\left(n-\frac{2 t}{n}\right)=1-\frac{2 t}{n^{2}}
$$

where $t$ is the number of alter-alter ties in ego's neighborhood and $n$ is the number of alters.

Assessing the dependence between message type and alter identity requires an index of dependence between variables $S$ (information type) and $I$ (alter ID) calculated separately for each value of $J$ (information receiver) - the ego. As the variables $S$ and $I$ are nominal, we need an index of stochastic dependence for which we choose the information-theoretic quantity (Lissowski 1977):

$$
\kappa \equiv \frac{H(S)-E(H(S \mid J))}{H(S)}
$$

where $H(\cdot)$ is the entropy of a, possibly conditional, distribution of a variable. The index varies between 0 (stochastic independence) and 1 (functional dependence), and has a convenient interpretation as a proportional reduction in prediction error (e.g. Agresti and Finlay 1997, chap. 8.7).

Figures 10.6 and 10.7 show actors in the Karate network plotted according to the strength of dependence between message type and alter ID (vertical axis) against two measures of brokerage (horizontal axis): Burt's constraint and network efficiency. In Figure 10.6 we see that actors with low values of constraint, the brokers, are characterized with higher dependence and the higher the constraint, the weaker the dependence. In Figure 10.7 the higher the network efficiency (indicating higher brokerage) the stronger the dependence. This leads us to:

Conjecture 1: (Brokers) In social networks actors in brokerage positions are characterized with a relatively strong dependence between (1) type of information arriving (identity of the seed node) and (2) an alter that is the source of the information for the actor.

\subsubsection{Ties}

We need to quantify two concepts: (1) the redundancy of alter $j$ to ego $i$ and (2) the extent to which the information coming from alter $j$ to ego $i$ is different from 


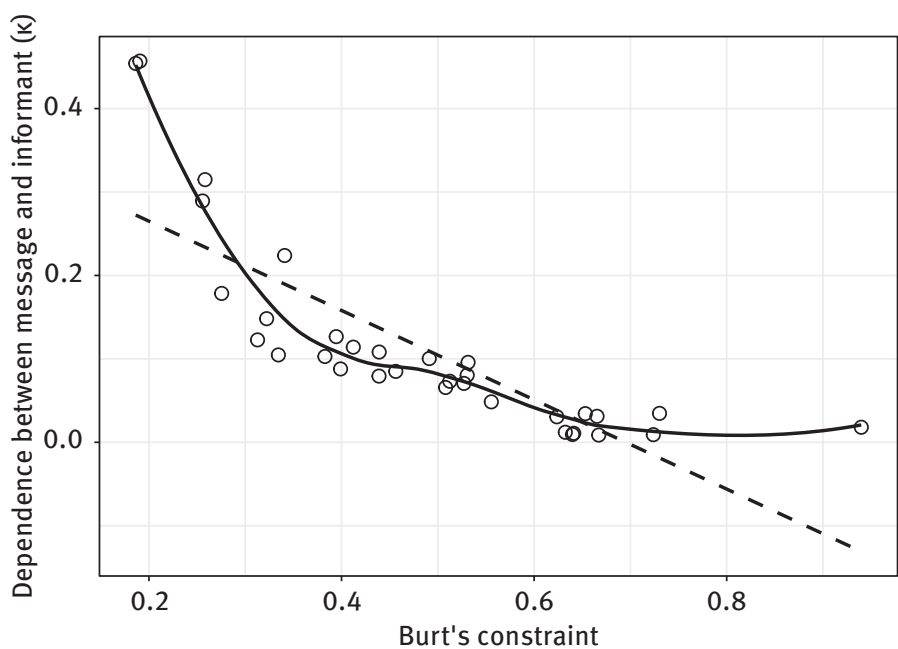

Figure 10.6: Strength of dependence between message type and informant versus Burt's constraint. For brokers (low constraint) it is more predictable what information comes from which alter. Linear (dashed) and LOESS (solid) trends superimposed.

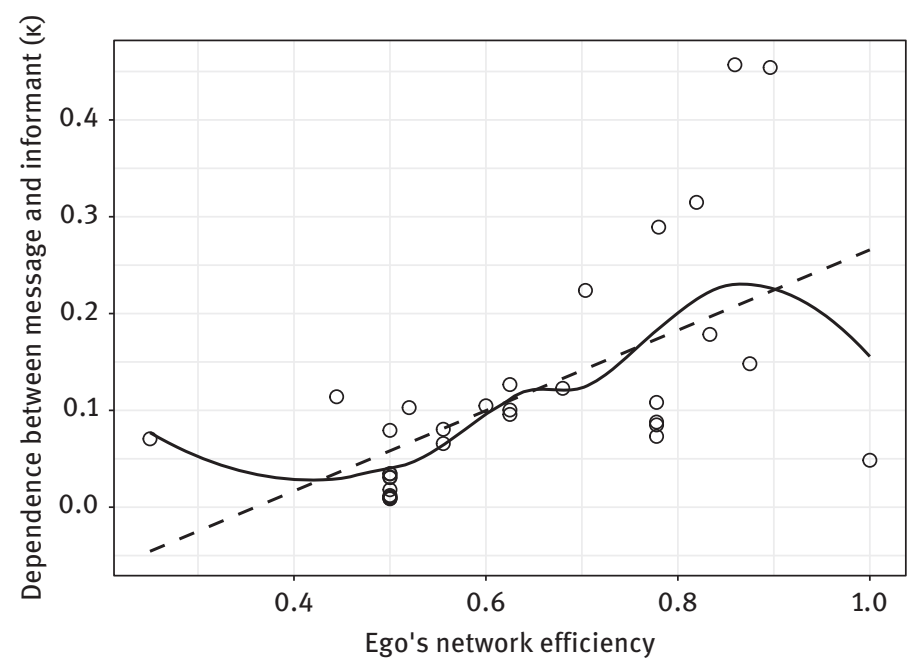

Figure 10.7: Strength of dependence between message type and informant versus ego's network efficiency. For brokers (high ego-network efficiency) it is more predictable what information comes from which alter. Linear (dashed) and LOESS (solid) trends superimposed.

information coming to ego from the remaining alters. Common way of measuring alter's redundancy is dyadic redundancy (Hanneman and Riddle 2005, chap. 9):

$$
\text { dyadic redundancy } y_{i j} \equiv \frac{d_{i j}}{n_{i}}
$$


where $d_{i j}$ is number of alter-alter ties in the neighborhood of ego $i$ adjacent to actor $j$ and $n_{i}$ is the number of alters of ego $i$.

To compare different distributions we will use Kullback-Leibler Divergence (Kullback and Leibler 1951; Kullback 1959). For two discrete probability distributions $p_{i}=P\left(X=x_{i}\right)$ and $q_{i}=P\left(Y=y_{i}\right)$ of some variables $X$ and $Y$ it is equal to

$$
\operatorname{KLD}(X, Y)=\sum_{i} p_{i} \log \frac{q_{i}}{p_{i}}
$$

It is equal to 0 if and only if distributions of $X$ and $Y$ are identical. In order to compare the distribution of information coming to ego from an alter to the distribution of information coming to ego from all other alters we are interested in calculating $\operatorname{KLD}(S|J=j \wedge I=i, S| J \neq j \wedge I=i)$.

Figure 10.8 shows all ego-alter ties in the Karate network according to dyadic redundancy (horizontal axis) and KL divergence calculated as above. We can see that alters characterized with low redundancy are more likely to bring different information to ego than those with high redundancy. This can be summarized as:

Conjecture 2: (Redundant ties) In personal social networks the two distributions of information types coming to an actor (1) from an alter and (2) coming from the remaining alters will be more different the lower the dyadic redundancy of that alter.

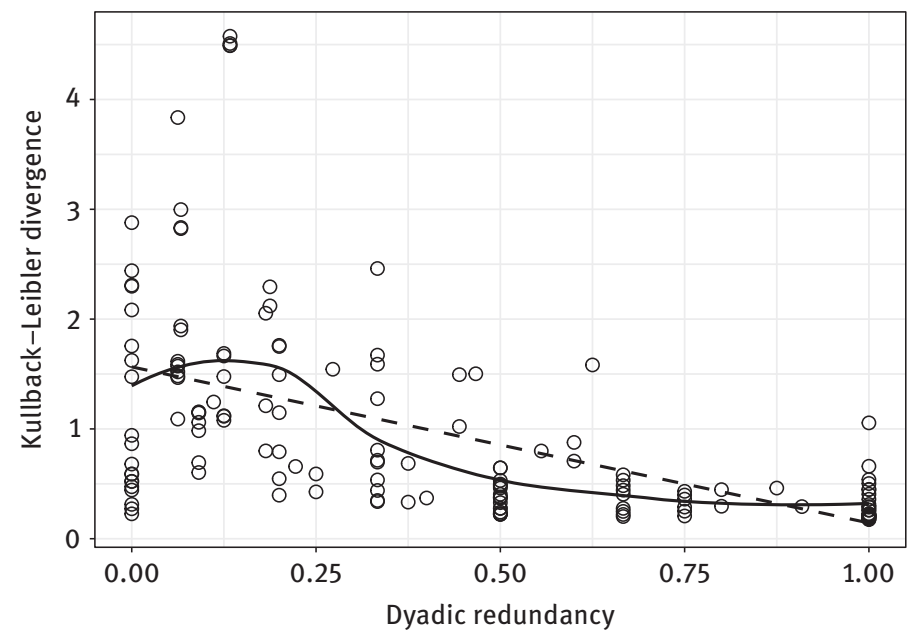

Figure 10.8: Dyad information divergence versus dyadic redundancy. Non-redundant ties (low dyadic redundancy) tend to bring different information than other ties. Linear (dashed) and LOESS (solid) trends superimposed.

Thus both conjectures are along the initial intuitions. Firstly, on the actor level, that brokerage positions are information-efficient. Secondly, on the tie level, that non-redundant ties tend to bring different information than other ties. 


\subsubsection{Triads}

We have argued in Section 10.2 that the consequences of occupying brokerage positions in a social network and information diffusion extend beyond the actor and dyadic levels. To make these intuitions more concrete we are going to analyze the simulated data on a triadic level by focusing on triples of actors consisting of ego $j$ and two alters $i$ and $k$ and the following properties:

1. The (dis)similarity between (a) distribution of information type that arrives to ego $j$ from alter $i$ and (b) distribution of information type that arrives to ego $j$ from alter $k$.

2. A measure capturing the extent of redundancy of alters $i$ and $k$ to ego $j$.

We will measure the dissimilarity between the distributions (item 1 above) with KullbackLeibler Divergence as defined in the previous section. To capture the triadic notion of redundancy (item 2) we may look at geodesic distance between alters $i$ and $k$ in ego's neighborhood. The closer the alters are the more they are redundant. Figure 10.9 shows all ego-alter-alter triplets for which the alter-alter distance is finite according to the alteralter distance (horizontal axis) and KL divergence measuring dissimilarity between information type distributions for the two alters in the triplet. We can indeed observe that the further the two alters are apart in the triplet the more dissimilar are the distributions of information type that arrive from these alters to ego. We summarize this with

Conjecture 3: (Alter-alter distance) In personal social networks the distributions of information types coming to the ego from two alters are the more dissimilar the greater the distance between these alters in ego's neighborhood.

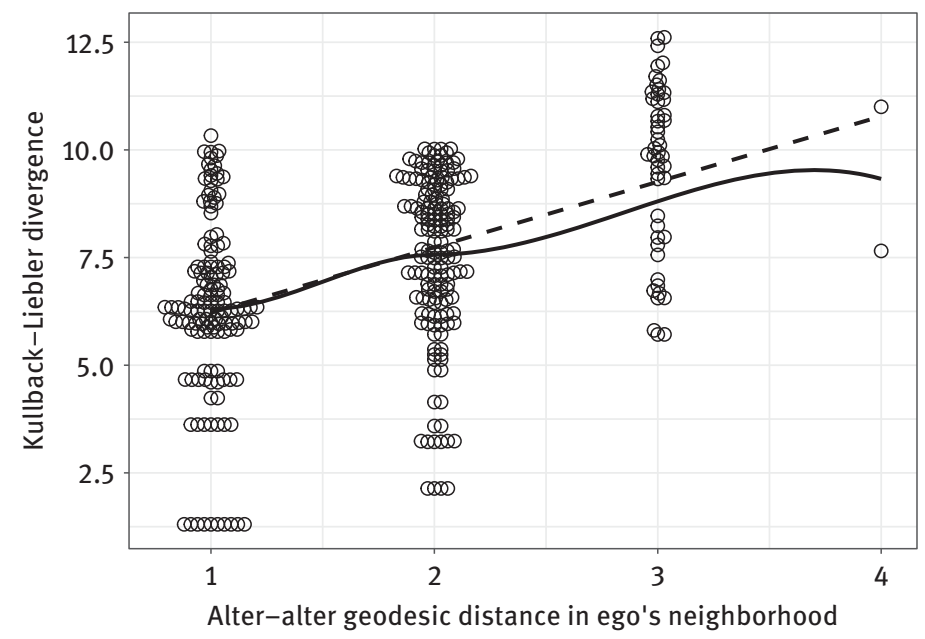

Figure 10.9: In ego-alter-alter triads in which alters are further apart, alters tend to bring more different information. Linear (dashed) and LOESS (solid) trends superimposed. 
Using alter-alter distance to indicate the extent of redundancy has two limitations. The first one is that we do not account for the pairs of alters who are disconnected. Distance between them is by convention equal to infinity. The second one is that we treat pairs of directly connected alters (at distance 1) as redundant to the same extent irrespectively of their embeddedness in the personal network of ego. To address the second issue we may capture that embeddedness by counting how many alters the pair of alters under study has in common. In the example network in Figure 10.1 actors $G$ and $H$ have no other alters in common while the pair $B-D$ has one alter in common - actor $C$. We might expect the more embedded a pair of alters is the more similar the distributions of information types will be. This is what we observe in Figure 10.10 which shows all ego-alter-alter triples in the Karate network according to the number of shared alters of the alter-alter pair (horizontal axis) and KL divergence of the distributions of information type coming from the two alters to ego. The more embedded the alter-alter pair is the less dissimilar the distributions. Hence the conjecture

Conjecture 4: (Alter-alter embeddedness) In personal social networks the distributions of information type coming to the ego from two alters are the less dissimilar the more embedded the alter-alter pair is in ego's neighborhood.

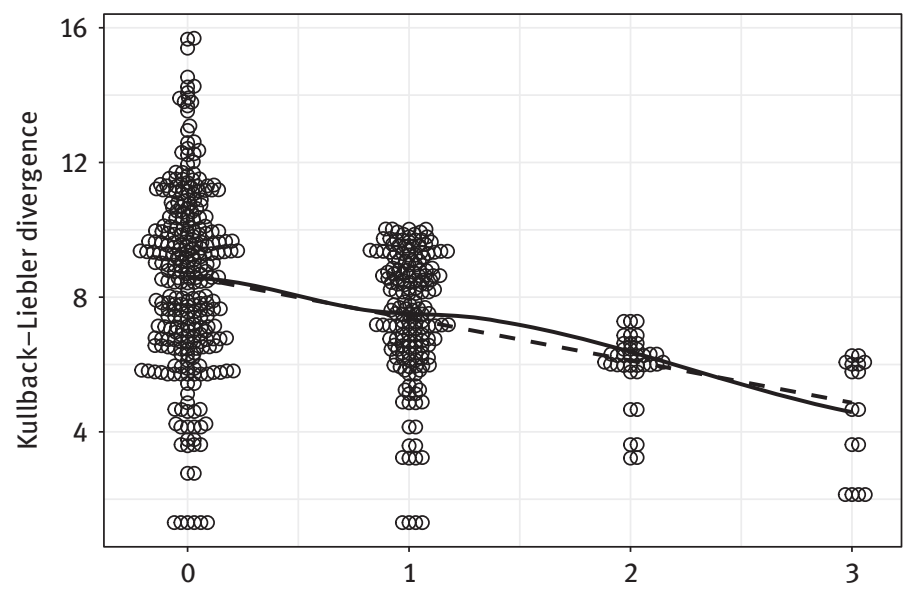

Number of partners shared by both alters in ego's neighborhood

Figure 10.10: In ego-alter-alter triads alters sharing more partners tend to bring similar information. Linear (dashed) and LOESS (solid) trends superimposed.

Table 10.1 shows a cross-classification of all ego-alter-alter triples in the Karate network. While number of shared partners (in columns) is shown as is, we have transformed the alter-alter distance (in rows) by (1) showing the inverse and (2) assigning value 0 to those triples in which the alters are not connected directly or indirectly. 
Notice that alter-alter distance and alter-alter embeddedness are almost complementary characteristics describing the neighborhood of of an ego. The (inverse) distance between the alters takes non-zero values only for those ego-alter-alter triples in which there are no shared partners indicating a null alter-alter embeddedness. This suggests that the two characteristics can be combined into a single numerical index.

Table 10.1: Cross-tabulation of inverse alter-alter distance and number of shared alters. The two measures are almost complementary.

\begin{tabular}{lrrrr}
\hline Inverse alter-alter distance & \multicolumn{4}{c}{ Number of shared alters } \\
\cline { 2 - 5 } & $\mathbf{0}$ & $\mathbf{1}$ & $\mathbf{2}$ & $\mathbf{3}$ \\
\hline 0.0000000 & 182 & 0 & 0 & 0 \\
0.2500000 & 2 & 0 & 0 & 0 \\
0.3333333 & 49 & 0 & 0 & 0 \\
0.5000000 & 0 & 154 & 2 & 4 \\
1.0000000 & 63 & 24 & 36 & 12 \\
\hline
\end{tabular}

Let $d(j, i, k)^{-1}$ be the inverse of the length of the shortest path between alters $i$ and $k$ in personal network of actor $j$ having all of $j$ 's ties removed. By convention we assume $d(j, i, k)^{-1}=0$ if the path $i$ and $k$ does not exist. Further, let $\operatorname{sh}(j, i, k)$ be the number of alters of ego $j$ who are directly connected to alters $i$ and $k$. The combined index, which we call pairwise redundancy (Bojanowski and Czerniawska-Szejda 2018) can be defined as:

$$
\operatorname{PR}(j, i, k)= \begin{cases}0 & \text { if } d(j, i, k)=0 \text { and } \operatorname{sh}(j, i, k)=0 \\ d(j, i, k)^{-1} & \text { if } d(j, i, k)>1 \text { and } \operatorname{sh}(j, i, k)=0 \\ 1 & \text { if } d(j, i, k)=1 \text { and } \operatorname{sh}(j, i, k)=0 \\ \operatorname{sh}(j, i, k)+1 & \text { if } d(j, i, k)=1 \text { and } \operatorname{sh}(j, i, k)>0\end{cases}
$$

In words:

- It is 0 if alters $i$ and $k$ of ego $j$ are not connected directly or indirectly, e.g. alter $F$ vs all other alters of ego $A$ in Figure 10.1.

- It is in the interval $(0,1)$ if alters $i$ and $k$ of ego $j$ are connected only indirectly, e.g. alters $B$ and $E$ of ego $A$ in Figure 10.1. It is the inverse of the shortest path between $j$ and $k$.

- It is 1 if alters $i$ and $k$ are connected directly with no shared partners, e.g. alters $G$ and $H$ of ego $A$ in Figure 10.1.

- It is in the interval $(1, \infty)$ if alters $i$ and $k$ are connected directly and have common some of ego's alters in common. It is the number of shared partners plus 1 . For example for alters $B$ and $D$ in Figure 10.1 it is $\operatorname{PR}(A, B, D)=1+1=2$ as alters $B$ and $D$ have alter $C$ in common. 
For illustration Table 10.2 contains all the pairwise redundancy scores for alters of ego $A$ in the network from Figure 10.1.

Table 10.2: Pairwise redundancy scores for pairs of alters of actor $A$ in the network from Figure 10.1.

\begin{tabular}{lllllll}
\hline Alter 1 & \multicolumn{5}{c}{ Alter 2 } \\
\cline { 2 - 7 } & C & D & E & F & G & H \\
\hline B & 2 & 2 & 0.5 & 0 & 0 & 0 \\
C & & 2 & 0.5 & 0 & 0 & 0 \\
D & & & 1 & 0 & 0 & 0 \\
E & & & & 0 & 0 & 0 \\
F & & & & & 0 & 0 \\
G & & & & & & 1 \\
\hline
\end{tabular}

We can now use the pairwise redundancy scores to characterize all ego-alter-alter triples from the Karate network jointly and set it against the KL divergence scores comparing the distributions of information type arriving from the alters to ego. This is shown in Figure 10.11. We can see that the more pairwise-redundant the alters are, the more similar the distributions of information type coming from these alters:

Conjecture 5: (Pairwise redundancy) In personal social networks the distributions of information type coming to the ego from two alters are the less dissimilar the more pairwise-redundant the alters are to ego.

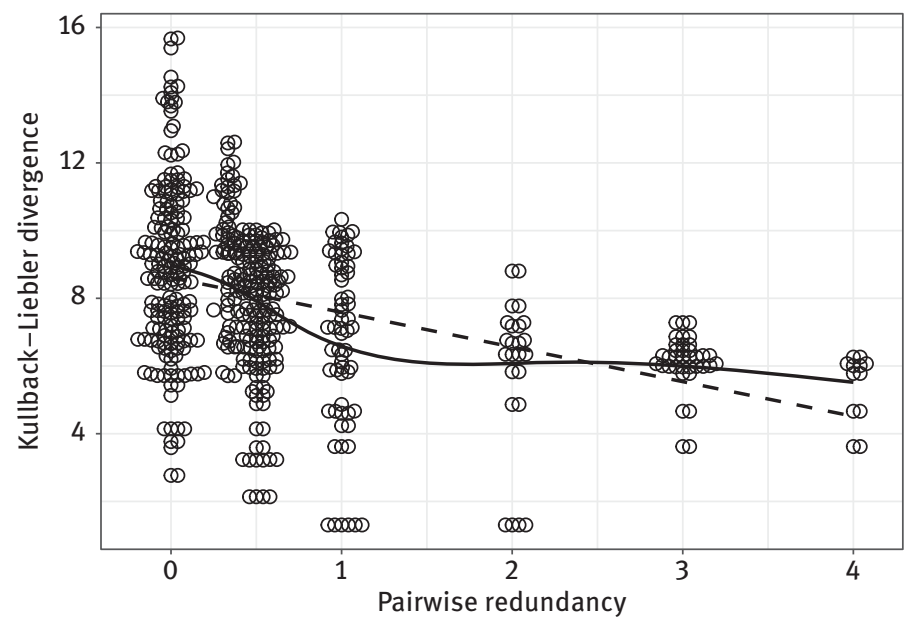

Figure 10.11: Pairwise-redundant alters tend to bring similar information. Linear (dashed) and LOESS (solid) trends superimposed. 
Conjectures 3, 4, and 5 formulated in this section are along the qualitative arguments discussed earlier, namely that there is a triadic dimension to brokerage and redundancy. Alters who might be redundant to the same extent may be at the same time non-redundant vis a vis each other in terms of the divergence of incoming information.

\subsection{Conclusion and discussion}

In the presented paper we have formalized a model of information diffusion and used computer simulation to show how statistical properties of information flow are related to brokerage characteristics on different levels of analysis. The results encapsulated in conjectures 1-5 provide us with viable empirical strategies for answering research questions regarding possible impact of the network structure on the tie content, if the latter is understood as the diversity of the types of information/resources being transferred or shared along that tie.

Consider a research setting in which there are multiple types of resources or information spreading in the network. Collected data could have a form of a set of dyadic variables, one for each resource, combined into an array, say, $R=\left[r_{i j k}\right]$. Elements of $R$ could be binary, e.g.: $r_{i j k}=1$ if resource $k$ has been shared/passed from actor $i$ to actor $j$ and $r_{i j k}=0$ otherwise. Alternatively $r_{i j k}$ 's could be frequencies of how often actor $j$ used resource $k$ provided by $i$ in some period of time. Symptoms of brokerage could be then analyzed on the three different levels we covered in the conjectures, namely:

- Node level: By Conjecture 1 we expect stronger dependence of information type and alter for brokers than for non-brokers. It is then sensible to characterize every actor $j$ with the strength of statistical association in the corresponding layers $r_{i(j) k}$. The actual choice of the measure of association depends on the measurement level of $r_{i j k}$ 's.

- Tie level: By Conjecture 2 we expect the non-redundant ties to be characterized with higher dissimilarity between distribution of incoming information types and the distribution of information incoming from other alters. Consequently, we may compare the dissimilarity of a bundle acquired by actor $j$ from actor $i$ described by the vector $r_{(i j) k}$ to aggregated bundles from all other neighbors of $j$.

- Triad level: By Conjectures 3, 4, and 5 we may compare resource bundles acquired by actor $j$ from actor $i$ to a resource bundle acquired by actor $j$ from some other actor $i^{\prime}$. If $r_{i j k}$ 's are binary a reasonable choice for dissimilarity measure is the Jaccard coeficient. The ego-alter-alter triads characterized with higher pairwise redundancy are then expected to be characterized with higher dissimilarity. An approach of this kind was used by Bojanowski and Czerniawska-Szejda (2018). 
A critical reader may notice that associations behind the formulated conjectures illustrated in Figures 10.6 through 11 show quite some variation. It is to be expected as our analyzes are based on personal networks of the actors. The choice was intentional as egocentrically sampled data is popular empirical material in the social sciences. The variation, at least in part, comes from higher-order structural properties of the network. In particular, higher-order redundancies. A natural follow-up research question could therefore be whether and how a concept similar to pairwise redundancy could be formulated when complete network data is available. An intuition already formulated by Burt (1995) points to ideas of "redundancy by equivalence".

More immediate problems are related to strengthening the presented results. Firstly, it should be possible to derive the conditional diffusion probabilities analytically. It seems promising to represent the information diffusion process formulated as a Markov chain in Section 10.3 terms of diffusion trees. The results are elusive though. Secondly, it is interesting whether the presented results will still hold under different variants of the information diffusion model. Obvious alternatives are the multinomial and product-binomial models of Buskens and Yamaguchi (1999). It seems that in the case of the multinomial model the differences should not be substantial. We leave these questions for further research.

\section{References}

Agresti, Alan, and Barbara Finlay. 1997. Statistical Methods for the Social Sciences. 3rd ed. New Jersey: Prentice Hall.

Bojanowski, Michal. 2019. Netflow: Simulating Network Flows (version of R package 0.0-1). https:// github.com/mbojan/netflow.

Bojanowski, Michat, and Dominika Czerniawska-Szejda. 2020. "Reaching for Unique Resources: Structural Holes and Specialization in Scientific Collaboration Networks." Journal of Social Structure 21(1): 1-34. https://doi.org/10.21307/joss-2020-001.

Borgatti, Stephen P. 2005. "Centrality and Network Flow." Social Networks 27 (1): 55-71.

Borgatti, Stephen P. 1997. "Structural Holes: Unpacking Burt's Redundancy Measures." Connections 20 (1): 35-38.

Burt, Ronald S. 1995. Structural Holes: The Social Structure of Competition. Cambridge, MA: Harvard University Press.

Burt, Ronald S., Robin M. Hogarth, and Claude Michaud. 2000. "The Social Capital of French and American Managers." Organizational Science 11 (2): 123-47. https://doi.org/10.1287/ orsc.11.2.123.12506.

Buskens, Vincent. 2002. Social Networks and Trust. Dordrecht: Kluwer.

Buskens, Vincent and Arnout van de Rijt. 2008. "Dynamics of Networks If Everyone Strives for Structural Holes.” American Journal of Sociology 114 (2): 371-407.

Buskens, Vincent, and Kazuo Yamaguchi. 1999. "A New Model for Information Diffusion in Heterogeneous Social Networks.” Sociological Methodology 29 (1): 281-325.

Csardi, Gabor, and Tamas Nepusz. 2006. "The Igraph Software Package for Complex Network Research.” InterJournal Complex Systems: 1695. http://igraph.org. 
Emerson, Richard M. 1962. "Power-Dependence Relations.” American Sociological Review, 27(1): 31-41.

Granovetter, Mark. 1974. Getting a Job: A Study of Contacts and Careers. Chicago: University of Chicago Press.

Granovetter, Mark S. 1977. "The Strength of Weak Ties." In Social Networks: A Developing Paradigm, edited by Samuel Leinhardt, 347-67. New York: Academic Press.

Hanneman, Robert A., and Mark Riddle. 2005. Introduction to Social Network Methods. University of California, Riverside. http://faculty.ucr.edu/ hanneman/.

Kullback, Solomon. 1959. Information Theory and Statistics. New York: John Wiley \& Sons.

Kullback, Solomon, and Richard Leibler. 1951. "On Information and Sufficiency." Annals of Mathematical Statistics 22 (1): 79-86.

Lissowski, Grzegorz. 1977. "Statistical Association and Prediction." In Problems of Formalization in the Social Sciences, edited by Klemens Szaniawski, 217-45. Wroctaw: Ossolineum.

Raub, Werner, Vincent Buskens, and Marcel Van Assen. 2011. "Micro-Macro Links and Microfoundations in Sociology." The Journal of Mathematical Sociology 35 (1-3): 1-25.

R Core Team. 2019. R: A Language and Environment for Statistical Computing (version 3.5.2). Vienna, Austria: R Foundation for Statistical Computing. https://www.R-project.org/.

Reagans, Ray, and Ezra Zuckerman. 2008. "Why Knowledge Does Not Equal Power: The Network Redundancy Trade-Off." Industrial and Corporate Change 17 (5): 903-44.

Schoch, David. 2019. Graphlayouts: Additional Layout Algorithms for Network Visualizations (version of R package 0.0.5.9000). https://github.com/schochastics/graphlayouts.

Zachary, Wayne W. 1977. "An Information Flow Model for Conflict and Fission in Small Groups." Journal of Anthropological Research 33 (4): 452-73. 